\title{
Wideband Optically-transparent Coatings Based on Composite Nanoscale Materials for the Optoelectronic Devices
}

\author{
Nicholas M. Ushakov ${ }^{1, *}$, Igor D. Kosobudsky ${ }^{1,2}$, Alexandr B. Zhimalov ${ }^{3}$, Pavel A. Muzalev ${ }^{1}$, Vitaly Ya. \\ Podvigalkin $^{1}$, Stanislav V. Eskin ${ }^{1,2}$, Dmitry M. Kulbatsky ${ }^{1}$ \\ ${ }^{1}$ Submicron Electronics Lab., SB Kotel'nikov IRE of RAS, Saratov, Russia \\ ${ }^{2}$ Saratov State Technical University, Saratov, Russia \\ ${ }^{3}$ Saratov Glass Institute, Saratov, Russia \\ *Corresponding Author: nmu@bk.ru
}

Copyright (C) 2013 Horizon Research Publishing All rights reserved.

\begin{abstract}
The paper presents two types of broadband antireflection coatings (AR) for silica glass in the range of optical wavelengths from $400 \mathrm{~nm}$ to $1000 \mathrm{~nm}$. Dense and porous AR coatings based on silica nanoparticles (NPs) of different structure were produced by sol-gel process. Thick film AR coating consisted of silver NPs in the matrix of poly(methyl methacrylate) (PMMA) was obtained on the substrate by liquid-phase deposition method. Synthesis conditions of the coatings materials of specified structure are revealed. The linear refraction and the linear absorption of these media at the different wavelength depending on the technology of preparation of coatings are analyzed. An increase in optical transmittance of silica glass with the coatings was up to $8-10 \%$ in comparison with the uncoated substrate.
\end{abstract}

Keywords Antireflection Coating, Fractal, Glass, Nanoparticle, Silica, Silver, Thick Film, Polymeric Matrix

\section{Introduction}

Shades with an AR coating are primary elements of the modern optoelectronic devices. They usually correspond to thin-film layered impedance transformers. Impedance value in the optical wavelength range can be controlled by changing refractive index and thickness of the certain layer. Thin-film AR coatings production technology is based, basically, on vacuum and ion-plasmatic methods [1]. Nanotechnology makes it possible to considerably simplify the coating process, increase transmission and decrease light reflection from glass surface in the wide range of optical wavelengths $[2,3]$.

In recent time there was interest in developing materials based on silica with controlled porosity. This parameter, in particular the pore size affects the refractive index, which allows to obtain materials with desired optical properties, in particular, anti-reflective coatings. Creating a porous antireflection coatings based on layers of nanoparticles (NPs) $\mathrm{SiO} 2$, with a loose packing, is a promising direction for research in connection with the possibility of obtaining composite glass with high light transmittance [4].

Furthermore, the polymer nanocomposite coating based on silver nanoparticles on glass and silicon substrates have a number of unique properties such as nonlinear optical properties [5] and giant photovoltaic effect [6]. Silver NPs have attracted interest due to their potential applications. Silver NPs have an advantage over other metal nanoparticles since the surface plasmon resonance energy of $\mathrm{Ag}$ is located far from the interband transition energy.

We consider the sol-gel technology for thin-films production on basis of $\mathrm{SiO} 2 \mathrm{NPs}$ [2] and the thick-film liquid-phase technology on basis of Ag NPs [3] as promising. These methods make it possible to produce coatings on substrates of almost any size - from the smallest (for integrated optical circuits) to the standard window size.

In this paper we present our studies of optical properties of nanocomposite coating on glass substartes based on $\mathrm{SiO} 2$ and Ag nanoparticles prepared by the sol-gel and the thick-film liquid-phase technology correspondingly. We analyze linear refraction and linear absorption of these media depending on the technology of preparation of coatings using the radiation of different wavelength.

\section{AR Coatings Based on Silica NPs}

\subsection{Experimental}

Both fractal and spherical silica NPs were synthesized by hydrolysis [equation (1)] of tetraethoxysilane [TEOS] (analytical grade, TU 2637-059-44493179-04) and subsequent condensation reactions [equations $(2,3)$ ] of 
intermediate species formed as a result of the hydrolysis.

$$
\begin{array}{r}
\equiv \mathrm{Si}-\mathrm{OC}_{2} \mathrm{H}_{5}+\mathrm{H}_{2} \mathrm{O}=\equiv \mathrm{Si}-\mathrm{OH}+\mathrm{C}_{2} \mathrm{H}_{5} \mathrm{OH}, \\
\equiv \mathrm{Si}-\mathrm{OC}_{2} \mathrm{H}_{5}+\mathrm{HO}-\mathrm{Si} \equiv=\equiv \mathrm{Si}-\mathrm{O}-\mathrm{Si} \equiv+\mathrm{C}_{2} \mathrm{H}_{5} \mathrm{OH}, \\
\equiv \mathrm{Si}-\mathrm{OH}+\mathrm{HO}-\mathrm{Si} \equiv=\equiv \mathrm{Si}-\mathrm{O}-\mathrm{Si} \equiv+\mathrm{H}_{2} \mathrm{O} .
\end{array}
$$

Ethanol $(\omega \approx 96 \%$, GOST $18300-87)$ was used as a solvent. For synthesis of the fractal NPs we used acetic acid (chemically pure, GOST 61-75) as a catalyst of the hydrolysis and the condensation reactions. Ammonia $[\omega=30-33 \%$, puriss, Classification according to Regulation (EC) No 1272/2008 (EU-GHS/CLP)] was used to catalyze the synthesis reactions of the spherical NPs. Molar ratios of the components were: TEOS/water/ethanol/acetic acid = 0.25/0.95/8.00/0.10 and TEOS/water/ethanol/ammonia $=$ $0.25 / 1.15 / 8.00 / 0.10$. During the synthesis the solutions were stirred in closed containers for $240 \pm 10 \mathrm{~min}$. Then they were aged for $168 \pm 1$ hours. Detailed guide of the synthesis is shown in many papers $[7,8]$.

AR coatings based on the silica NPs on silica glass were obtained by dip coating process. Withdrawal speed of the substrate was $150 \pm 5 \mathrm{~mm} / \mathrm{min}$. After that the coated glasses were heat-treated at $500 \pm 5^{\circ} \mathrm{C}$ for $15 \pm 5 \mathrm{~min}$.

Shape and mean diameter of the spherical NPs were studied by scanning electron microscope (SEM) MIRA II (Tescan) and Zetasizer Nano ZS analyzer (Malvern Instruments Ltd). Fractal dimension $\left(d_{f}\right)$ and radius of gyration $\left(R_{g}\right)$ measurements by small-angle X-ray scattering (SAXS) are still in progress since it needs many samples to average data. However, we've got preliminary results already.

The coatings thickness was measured by Dektak 150 surface profiler (we scratched the coatings before thermal treatment by ABS plastic and then calculated depth of the scratch).

Refractive index of the coatings (and their porosity) and glass was obtained by ellipsometry (Ellipse-1000 ASG, Spectran software). If $d_{\text {mean }}<<\lambda$ in the electrostatic approximation, where $d_{\text {mean }}$ - mean diameter of the spherical NPs, effective permittivity of the heterostructure (spherical $\mathrm{NPs}+$ air) is described by Bruggeman model (effective medium approximation):

$$
p\left(\frac{\varepsilon_{p}(\lambda)-\varepsilon_{e f f}(\lambda)}{2 \varepsilon_{e f f}(\lambda)+\varepsilon_{p}(\lambda)}\right)+(1-p)\left(\frac{\varepsilon_{m}(\lambda)-\varepsilon_{e f f}(\lambda)}{2 \varepsilon_{e f f}(\lambda)+\varepsilon_{m}(\lambda)}\right)=0,
$$

where $p$ - porosity, $\varepsilon_{p}$ - permittivity of air (for dry air $\varepsilon_{p}=1$ ), $\varepsilon_{m}$ - permittivity of the matrix (silica), $\varepsilon_{m}=n_{\text {eff }}^{2}$ effective permittivity of the porous coating [9]. This model is appropriate for describing a porous coating optical behavior. Meanwhile, the model of thin solid film was used for description of the dense coating based on the fractal NPs.

Optical transmittance of the samples was measured by Lambda 950 spectrophotometer within the wide range of wavelengths $(300-2000 \mathrm{~nm})$ with the step of $10 \mathrm{~nm}$.

\subsection{Results and Discussion}

From the results of measurements of refractive indexes of the materials by ellipsometry we've found out that the coating consisted of the fractal NPs was dense [see figure 1 (a)] (thickness obtained by ellipsometry $\mathrm{d}=90 \pm 3 \mathrm{~nm}$ ) and the coating based on spherical NPs [see figure 1 (b)] ( $d=134 \pm 4$ $\mathrm{nm})$.

Calculated from the Bruggeman equation porosity of the coating based on spherical NPs was about $44 \pm 2 \%$. This was due to spherical shape of the NPs, which were loosely packed within the coating.

SAXS showed that the fractal NPs had $\mathrm{df} \approx 1.5$ and $\mathrm{Rg}$ of approximately $10 \mathrm{~nm}$. Such tiny structures were densely packed together within the coating due to their shape and dimensions.

Due to the almost complete lack of porosity of this material, the shrinkage of the coating was caused by polycondensation reactions $(2,3)$ and structural relaxation [10]. Therefore hardening of the coating was due to formation of new siloxane bonds $\equiv \mathrm{Si}-\mathrm{O}-\mathrm{Si} \equiv$, as well as structural relaxation - irreversible process, in which the free energy decreases due to the restructuring of the chemical bonds without mass loss [11]. Fig. 2 (a) shows a photograph (SEM) of the coating (top view), which had visible defect in its structure - the groove.

Perhaps it was formed during the thermal treatment due to removal of volatile molecules from the coating (ethanol, water, etc.). In general, the coating had uniform structure with a small number of such defects. Importantly, adhesion of the coating to the substrate was increased after the thermal treatment, which was probably due to reactions $(2,3)$, i.e. the formation of siloxane bonds between the particles of the coating and the glass. Since it is known that the surface structure of silicate glass and silica particles is similar - in both cases different types of silanol groups are presented $[10]$. Moreover, according to $[12,13]$ silanol number of these materials is almost the same $(4.6-\mathrm{OH} / \mathrm{nm} 2)$.

Shinkage of the porous coating, consisting of spherical silica particles during the thermal treatment was caused mostly by desorption of ethanol, water and catalyst molecules. According to [10] in this case capillary contraction takes place. Desorption of the volatile molecules from the surface of the silica particles increases their surface energy, and hence the pressure in the capillary pores. Since the thermal treatment temperature is close to glass transition temperature of silica glass [13], further shrinkage and compaction occur due to viscous sintering. This process occurs due to the energy obtained by decreasing the surface area of the porous body.

Fig. 2 (b) shows a photograph (SEM) of coatings based on spherical silica particles. It is clearly seen that the coating is consisted of sintered spherical particles having an average diameter of about $100 \mathrm{~nm}$ and quite low polydispersity index (PDI) - 0.091 (which was measured in sol by dynamic light scattering method).F 


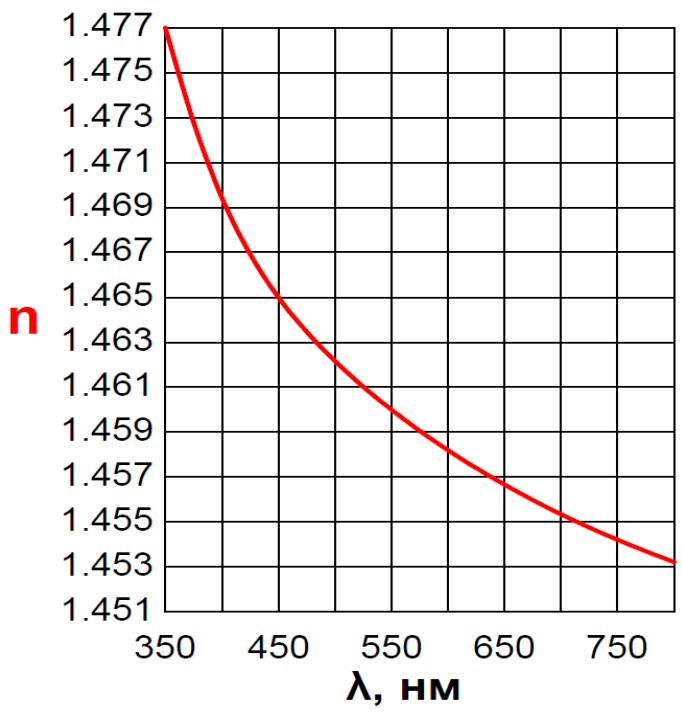

(a)

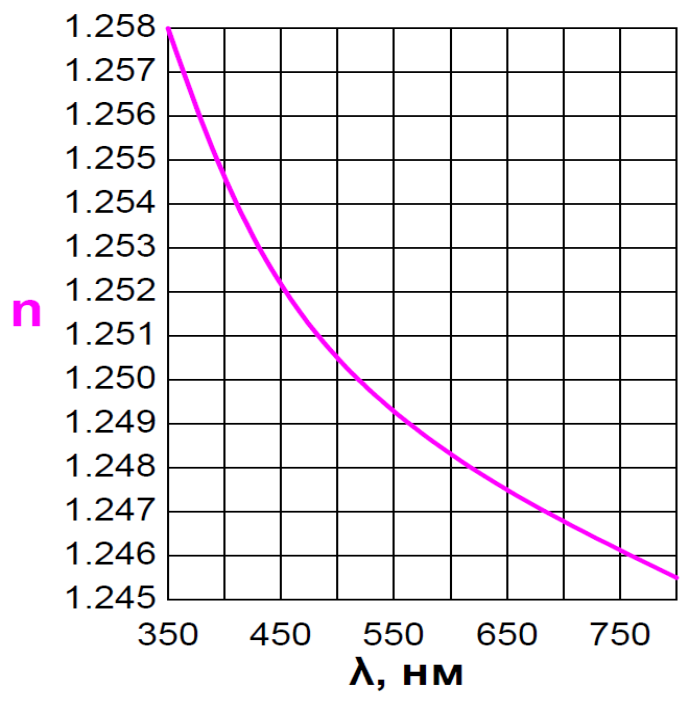

(b)

Figure 1. Refractive index of dense coating (a) and effective refractive index of porous coating (b)

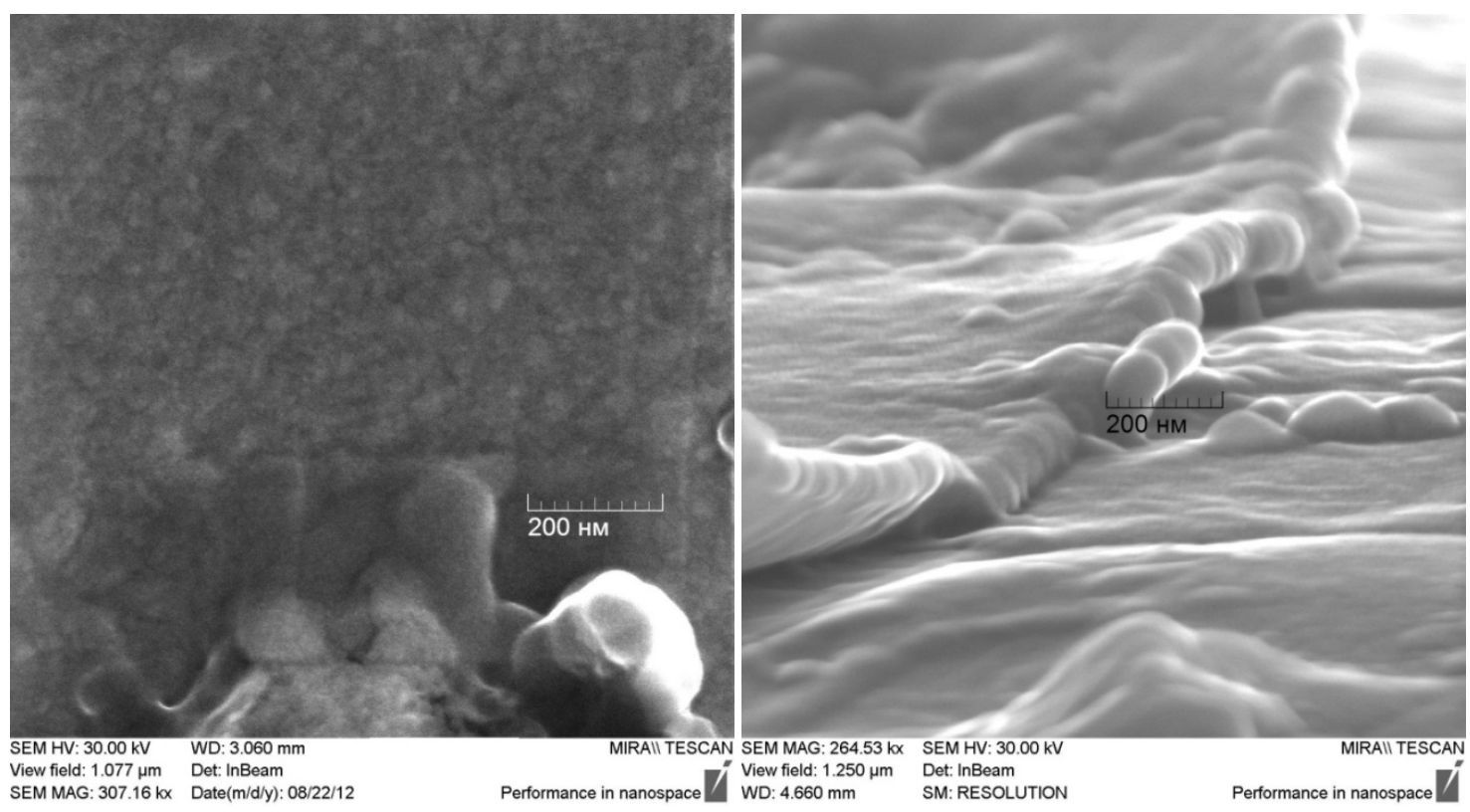

(a)

(b)

Figure 2. SEM images of dense coating (a) and the porous one (b)

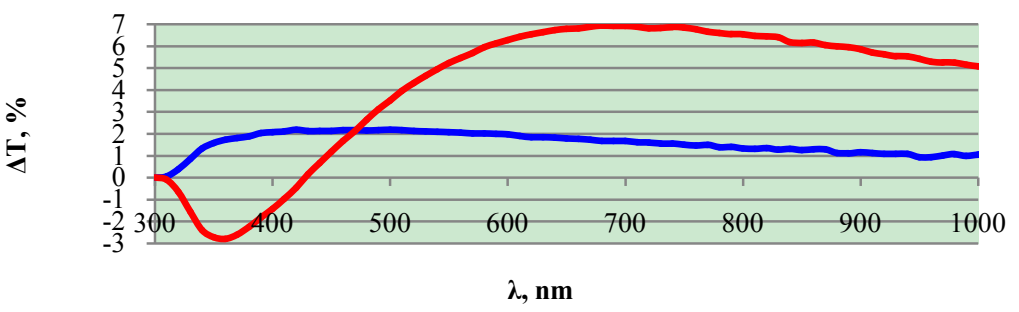

Glass with the dense AR coating

Glass with the porous AR coating

Figure 3. Change in optical transmittance of silica glass with the AR coatings in comparison with the uncoated glass. $\Delta \mathrm{T}=\mathrm{Tglass}$ with the $\mathrm{AR}$ coating Tglass without the AR coating. 
Thickness of the dense and porous coatings measured by the profiler was $90 \pm 4 \mathrm{~nm}$ and $131 \pm 10 \mathrm{~nm}$, respectively.

Fig. 3 shows change in optical transmittance of the glass with the AR coatings (on both surfaces of the substrate) in comparison with the uncoated.

The porous AR coating is more effective than the dense one and allows increasing optical transmittance of silica glass by almost $7 \%$ due to its low effective refractive index. Thus it better fits the AR condition that refractive index of an AR coating should be square root of product of substrate and incidence medium refractive indexes (for silica glass $\mathrm{n} \approx 1.52$ at $\lambda=550 \mathrm{~nm}$; for dry air $\mathrm{n} \approx 1.00$ at $\lambda=550 \mathrm{~nm}$ ).

Despite of better optical behavior, the porous coating has lower abrasion resistance than the dense one. That's why the porous one is reasonable to use in inner optics (multiple-lens objectives etc.). The dense coating has lower AR effect but high toughness (almost like the glass one). Silica glass with this AR coating can be successfully used for various displays and solar photoelectric transducers as a protective element.

\section{Thick Film AR Coating}

\subsection{Experimental}

Silver NPs were synthesized by reduction of metal salt $\left(\mathrm{Ag}(\mathrm{NO})_{3}\right.$, reagent grade, GOST 1277-75) in polymeric matrix (PMMA, Dakril-8, TU 2216-243-05757593-2000) (thermal decomposition method [14]).

The polymer solution was prepared in mineral oil (VM-1, NTD-6). Ethylene chloride (chemically pure, GOST 1942-86) was used as a solvent of PMMA. Then the metal salt was dissolved in excess of ammonia $(\omega=25 \%$, reagent grade, GOST 3760-79) with the help of magnetic stirrer to obtain the silver-containing precursor. A reactor (round-bottom flask containing the polymer solution) was filled with inert gas (argon) which was preliminary purified from oxygen and nitrogen impurities. After that the polymer solution in mineral oil was heated up to $200-250{ }^{\circ} \mathrm{C}$ under continuing stirring by overhead stirrer (250-400 rpm). Then the precursor and the reduction agent (hydrazine hydrate, $2 \times 10^{-2}$ ) were added dropwise to the polymer solution. The mixture was sonicated during the stirring ( $v=25 \mathrm{kHz}, P=75$ $\mathrm{W})$. After that the mixture was extracted from mineral oil by hexane (chemically pure, GOST 2631-98) and dried in a heat chamber for 48 hours $\left(\mathrm{T}=50{ }^{\circ} \mathrm{C}\right)$. Obtained powders had different color depending on the metal concentration which varied from 1 to 10 weight percent.

Thick films of the composite material (silver NPs in PMMA) were obtained on one surface of silica glass (which was preliminary etched by $\mathrm{NaOH}$ water solution to enhance the film adhesion). For it the dissolved in ethylene chloride composite was flew on the substrate and smoothed by a squeegee. Then the films were dried on air for one day. The presented coating process takes a little time (no more than 20 minutes). That's why it's possible to prevent polymeric suspension coagulation. It's also possible to automate this process.
The physicochemical properties of the composites produced were investigated by a number of optical spectroscopy methods, electron microscopy, and X-ray diffraction analysis (XPD).

The composition and structure of the metal-containing nanocomposite were studied by XPD (DRON-4, Russia) using an X-ray tube with a copper anode $(\mathrm{CuK} \alpha$ irradiation, $\lambda=0.154056 \mathrm{~nm})$.

\subsection{Results and Discussion}

Stabilized in PMMA silver NPs can be seen in Figure 4. In most cases spherical silver NPs with a mean size $d=20-40 \mathrm{~nm}$ were formed in the polymeric matrix.

It was important to find out the optimal metal concentration in the polymeric matrix experimentally at which maximum AR effect of the coating would be observed.

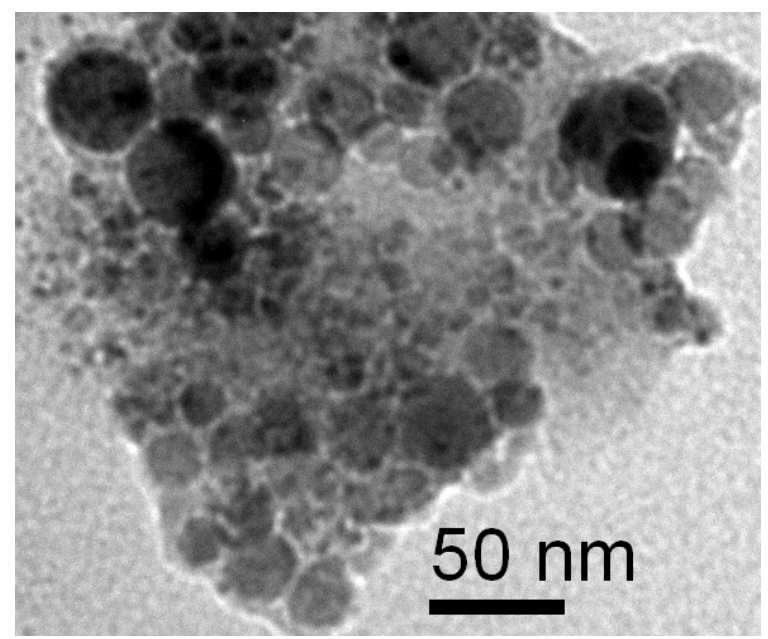

Figure 4. Scanning electron micrograph of silver NPs in PMMA.

In fig. 5 a ratio of the coated silica glass reflection coefficient to uncoated silica glass reflection coefficient is presented. It can be clearly seen that AR coating reduced reflectance from silica glass approximately by $10 \%$ in the range of wavelengths from 400 to $850 \mathrm{~nm}$.

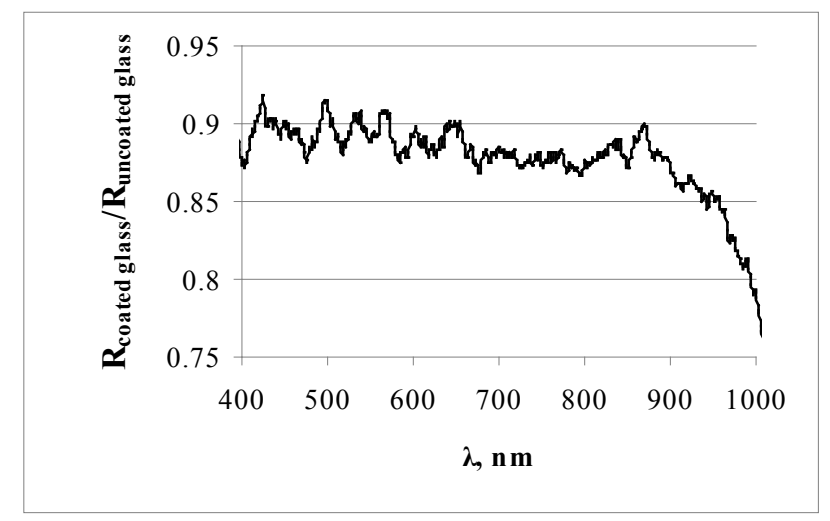

Figure 5. A ratio of the coated silica glass (PMMA+Ag [3 wt.\%] thick film) reflection coefficient to uncoated substrate reflection coefficient depending on $\lambda$. Film thickness - $35 \mu \mathrm{m}$. 
A ratio of the coated silica glasses transmission coefficient to uncoated silica glass transmission coefficient is shown in fig. 6. It can be seen that optical properties of the thick film AR coating depend on its thickness. A maximum increase in glass transmittance almost by $15 \%$ in visible range of wavelengths was observed for the coating which had thickness of $80 \mu \mathrm{m}$.

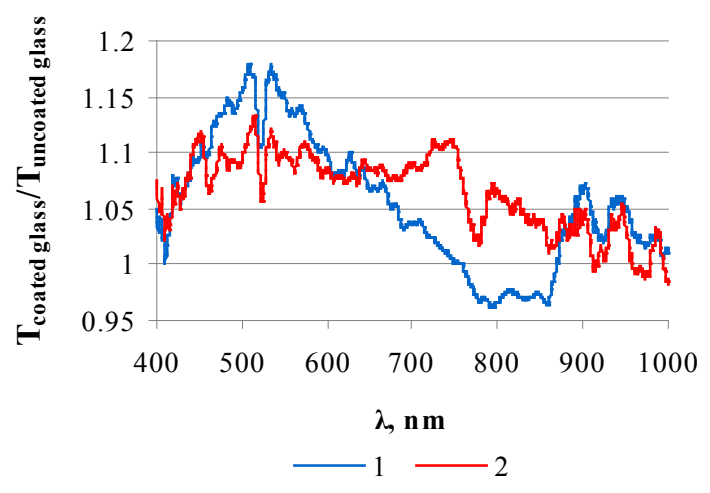

Figure 6. A ratio of the coated silica glasses (PMMA+Ag [3wt.\%] thick films) transmission coefficient to uncoated silica glass transmission coefficient depending on $\lambda$. Film thickness: $1-35 \mu \mathrm{m}, 2-80 \mu \mathrm{m}$.

The thick-film coating corresponds to heterogeneous disordered two-component medium. It has refractive index less than 1. Such composite nanomaterial is consisted of disordered 3D array of silver nanoantennas with dimensions of 30-40 nm. Two adjacent silver nanoparticles can be described as dipole nanoantenna with clearance with dimensions of 1-2 of nanoparticle diameter. Local fields are being increased $4-5$ and more orders in nanoclearance (Purcell factor). It causes some interesting physical phenomena such as gigantic photoelectric effect, gigantic combinational scattering and others. Our technology makes it possible to create ordered structures with uniform clearance unlike disordered structures. In this case nanoantennas correspond to multiple Yagi-Uda antenna. These antennas can be joined with quantum dots based on various materials (near-field communication). Plasmon resonance appears (synchronous oscillation of electrons on the silver nanoparticles surface). That improves efficiency of the system and transform quantum dot to directional emitter.

\section{Conclusion}

Two types of AR coatings were obtained on silica glass and studied in detail.

The first type corresponds to a quarter-wave single layer AR coating. Such coatings based on fractal and spherical silica NPs allowed to increase silica glass transmittance by almost 2.5 and 7 percent, respectively. The porous coating (consisted of spherical NPs) was more efficient than the dense one due to its low effective refractive index $\left(n_{\text {eff }} \approx 1.28\right.$ at $\lambda=550 \mathrm{~nm}$ ). However, the porous coating had very low adhesion to the substrate in comparison with the dense one.
That's why the coating based on the spherical NPs can be used in inner optics. On the contrary silica glass with the dense AR coating is appropriate to use as a protective element for solar cells, various displays etc. Moreover, manufacturing of the mentioned above coatings is quite simple and low-cost.

The second type of match in thick (up to 100 microns) polymer composite coating based on silver nanoparticles in the matrix of polymethylmethacrylate. Silver nanoparticles as the nanoantenna array with the absorption of optical radiation at a wavelength of $380-400 \mathrm{~nm}$ (surface plasmon resonance), and amplified reemission at higher wavelengths (more $500 \mathrm{~nm}$ ). This phenomenon allows the use of such coatings as active coatings for glass and a number of optoelectronic devices, including photovoltaic cells.

\section{Acknowledgements}

This study was financially supported by Russian Foundation for Basic Research (grant \# 11-08-00351-a).

\section{REFERENCES}

[1] A. Thelen. Equivalent layers in multilayer filters, Journal of Opt. Soc. Am., Vol. 56, No. 6, pp. 1533-1538, 1966. doi:10.1364/JOSA.56.001533

[2] S. V. Eskin, I. D. Kosobudsky, A. B. Zhimalov, N. M. Ushakov, D. M. Kulbatsky and S. V. German. Antireflection coating based on $\mathrm{SiO} 2$, Journal of Inorganic Materials, Vol. 48, No. 10, pp. 1133-1138, 2012.

[3] P. A. Muzalev, I. D. Kosobudsky, D. M. Kulbatsky and N. M. Ushakov. Polymer composite materials based on PMMA with silver nanoparticles, the synthesis and optical properties, Journal of Material Science, No. 5, pp. 18-21, 2011.

[4] Braun M.M., Pilon L. Effective optical properties of non-absorbing nanoporous thin films, Journal of Thin Solid Films, Vol. 496, pp. 505-514, 2006.

[5] R.A. Ganeev, M. Baba, A.I. Ryasnyansky, M. Suzuki, and H. Kuroda. Characterization of optical and nonlinear optical properties of silver nanoparticles prepared by laser ablation in various liquids, Journal of Optics Communications, Vol. 240, pp. 437-448, 2004.

[6] O.N. Gadomsky, K.K. Altunin, N.M. Ushakov, D.M. Kul'backii. Giant photovoltaic effect, Journal of Pis'ma v ZhETF, Vol. 93, iss. 6, pp.353-358, 2011.

[7] B. Karmakar and D. Ganguli. Dense silica microspheres from organic and inorganic acid hydrolysis of TEOS, Journal of Non-Crystalline Solids, Vol. 272, No. 2-3, pp. 119-126, 2000. doi:10.1016/S0022-3093(00)00231-3

[8] G. H. Bogush, M. A. Tracy and C. F. Zukovski. Preparation of Monodisperse Silica Particles: Control of Size and Mass Fraction, Journal of Non-Crystalline Solids, Vol. 104, No. 1, pp. 95-106, 1988. doi:10.1016/0022-3093(88)90187-1

[9] J. Zhenhong. Determination of the effective refractive index 
of porous silicon/polymer composite films, Journal of Chinese Optics Letters, Vol. 3, No. 10, pp. 608-610, 2005.

[10] Brinker C.J. Sol-Gel Science the Physics and Chemistry of Sol-Gel Processing, San Diego: Academic Press, Inc., 908 p, 1990.

[11] Scherer G.W. Relaxation in Glass and Composites, New York: Wiley, 331 p, 1986.
[12] Zhuravlev L.T. The surface chemistry of amorphous silica. Zhuravlev model, Colloids and Surfaces A: Physicochemical and Engineering Aspects, Vol. 173, No 1-3, pp. 1-38, 2000.

[13] Le Bourhis E. Glass Mechanics and Technology, Weinheim: WILEY-VCH Verlag GmbH \& Co. KGaA, 366 p., 2008.

[14] S. P. Gubin. Single-phase metallopolymers, DAN USSR, Vol. 273, No. 5, pp. 1155-1158, 1983. 